\title{
Relationship between Skeletal Muscle Mass, Bone Mineral Density, and Trabecular Bone Score in Osteoporotic Vertebral Compression Fractures
}

\author{
Soichiro Tokeshi ${ }^{1}$, Yawara Eguchi ${ }^{2}$, Munetaka Suzuki ${ }^{1}$, Hajime Yamanaka ${ }^{1}$, \\ Hiroshi Tamai ${ }^{1}$, Sumihisa Orita ${ }^{2}$, Kazuhide Inage ${ }^{2}$, Yasuhiro Shiga ${ }^{2}$, Shigeo Hagiwara ${ }^{2}$, \\ Junichi Nakamura ${ }^{2}$, Tsutomu Akazawa ${ }^{3}$, Hiroshi Takahashi ${ }^{4}$, Seiji Ohtori ${ }^{2}$ \\ ${ }^{1}$ Department of Orthopaedic Surgery, Shimoshizu National Hospital, Yotsukaido, Japan \\ ${ }^{2}$ Department of Orthopaedic Surgery, Graduate School of Medicine, Chiba University, Chiba, Japan \\ ${ }^{3}$ Department of Orthopaedic Surgery, St. Marianna University School of Medicine, Kawasaki, Japan \\ ${ }^{4}$ Department of Orthopaedic Surgery, Toho University Sakura Medical Center, Sakura, Japan
}

Study Design: A retrospective observational study was performed.

Purpose: We investigated the relationships between skeletal muscle mass, bone mineral density (BMD), and trabecular bone score (TBS) in patients with osteoporotic vertebral compression fractures (VCFs).

Overview of Literature: The TBS has attracted attention as a measurement of trabecular bone microarchitecture. It is derived from data obtained using dual-energy X-ray absorptiometry (DXA) and is a reported indicator of VCFs, and its addition to the Fracture Risk Assessment Tool increases the accuracy of fracture prediction.

Methods: BMD, skeletal muscle mass, and TBS were measured in 142 patients who visited Shimoshizu National Hospital from April to August 2019. Patients were divided into a VCF group and a non-VCF group. Whole-body DXA scans were performed to analyze body composition, including appendicular skeletal muscle mass index (SMl; lean mass $[\mathrm{kg}] /$ height $\left[\mathrm{m}^{2}\right]$ ) and BMD. The diagnostic criteria for sarcopenia was an appendicular $\mathrm{SMI}<5.46 \mathrm{~kg} / \mathrm{m}^{2}$. A logistic regression analysis was conducted to identify the risk factors for VCFs. Results: The significant $(p<0.05)$ findings (VCF group vs. non-VCF group, respectively) included age (79 vs. 70 years), femoral BMD $\left(0.50\right.$ vs. $\left.0.58 \mathrm{~g} / \mathrm{cm}^{2}\right)$, TBS (1.25 vs. 1.29), and lower limb muscle mass ( $\left.8.6 \mathrm{vs.} 9.9 \mathrm{~kg}\right)$. The VCF group was significantly older and had a lower femur BMD and decreased leg muscle mass than the non-VCF group. Based on the multiple logistic regression analysis, lower femoral BMD and decreased leg muscle mass were identified as risk factors for vertebral fracture independent of age, but the TBS was not.

Conclusions: Patients with VCFs had low BMD, a low TBS, and low skeletal muscle mass. Lower femoral BMD and decreased leg muscle mass were identified as risk factors for VCFs independent of age, whereas the TBS was not identified as a risk factor for VCFs.

Keywords: Osteoporosis; Spinal fractures; Bone density; Skeletal muscle

\footnotetext{
Received Feb 4, 2020; Revised Mar 20, 2020; Accepted Apr 1, 2020

Corresponding author: Yawara Eguchi

Department of Orthopaedic Surgery, Center for Orthopaedic Science Medical Innovation, Graduate School of Medicine, Chiba University, 1-8-1 Inohana, Chuo-ku, Chiba, 260-8670, Japan

Tel: +81-43-226-2117, Fax: +81-43-226-2116, E-mail: yawara_eguchi@yahoo.co.jp
} 


\section{Introduction}

Osteoporotic vertebral compression fractures (VCFs) are common in the elderly and cause gait disturbances and reduce activities of daily living. Sakuma et al. [1] showed that $81 \%$ of patients with a hip fracture had also sustained a vertebral fracture. Moreover, the average age at the time of injury was higher for hip fractures (81.4 years) than for vertebral fractures (77.7 years). Vertebral fractures can lead to hip fractures; therefore, early strategies aimed at fracture prevention are important, particularly for elderly patients with osteoporosis [1].

The trabecular bone score (TBS) estimates the threedimensional microarchitectural characteristics of lumbar vertebrae. This is achieved via analysis of two-dimensional lumbar images acquired using variogram-based assessments of dual-energy X-ray absorptiometry (DXA) scans to determine bone mineral density (BMD). Because significant correlations have been demonstrated in the spine between the TBS and the parameters of trabecular connectivity density, bone volume fraction, and trabecular number, bone microarchitecture can be evaluated $[2,3]$.

The TBS is an independent predictor of fracture risk in patients with primary osteoporosis $[2,4,5]$. In contrast, the TBS has been reported to correlate with fracture risk better than bone density in patients with secondary osteoporosis due to steroid therapy [6-8], rheumatoid arthritis $[8,9]$, and type 2 diabetes mellitus $[10,11]$, and this propensity has attracted attention in recent years.

Sarcopenia is a syndrome characterized by a progressive, generalized loss of skeletal muscle mass and skeletal muscle strength [12]. People with sarcopenia are predisposed to a higher than normal risk of falling and becoming bedridden, which can cause significant physical and economic loss to a society with an increasing proportion of elderly people in the population. Sarcopenia, osteoporosis, and degenerative scoliosis have been reported to be interrelated in middle-aged women $[13,14]$. Eguchi et al. [15] reported that decreased skeletal muscle mass may cause scoliosis, anteversion, and pelvic retroversion that results in spinal deformity and back pain; therefore, the treatment and prevention of sarcopenia is critical. Reduced leg muscle mass and poor grip strength are also risk factors for the development of VCFs, which may be profoundly implicated in the pathogenesis of sarcopenia [16]. However, there are no reports on the relationships between VCFs, sarcopenia, and bone microarchitecture.
In this study, we examined the relationships between skeletal muscle mass, BMD, and TBS and VCFs.

\section{Materials and Methods}

\section{Participants}

We declare that all protocols involving humans have been approved by the Shimoshizu National Hospital and have been performed in accordance with the ethical standards laid down in the 1964 Declaration of Helsinki and its later amendments. Informed consent was obtained from all participants before the study began. The study protocol was approved by an ethical review committee (IRB approval no., H26'-6).

A total of 169 patients attended orthopedic outpatient clinic visits from April to August 2019. After excluding 27 patients with implants, whole-body BMD, whole-body skeletal muscle mass, and TBS measurements with DXA were evaluated in the remaining patients. A total of 142 participants (17 men, 125 women; mean age, $74.6 \pm 8.6$ years; range, $44-96$ years) were included. The population sample was divided into two groups: 43 participants who had osteoporosis-related thoracolumbar fractures (VCF group; three men, 40 women; mean age, $79 \pm 6.8$ years) and 99 participants without compression fractures (non-VCF group; 14 men, 85 women; mean age, $70 \pm 9.9$ years) (Table 1 ).

\section{Radiographic analysis}

Lateral view radiographs of the lumbar spine were performed with the patient in a standing position. VCFs were defined as wedge, biconcave, and compound fractures based on the images (Fig. 1), which revealed the dimensions of the vertebral body, and the diagnostic criteria issued by the Ministry of Health and Welfare [17]. It was

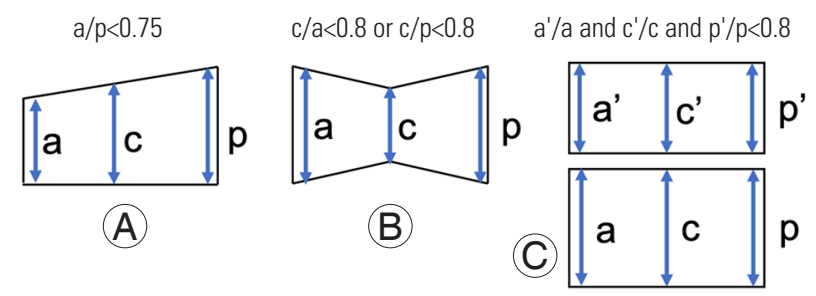

Fig. 1. Diagnostic criteria for vertebral fractures. Vertebral compression fractures in lateral spinal radiographs were defined as either wedge (A), biconcave (B), or compound (C). 
challenging to identify new vertebral fractures. Patients who visited the hospital for symptoms such as new onset back pain and were diagnosed with a vertebral fracture by an orthopedic surgeon based on a radiographic and physical examination were considered to be new fracture cases (i.e., clinical fractures). Asymptomatic older fractures (i.e., prevalent fractures) discovered incidentally using radiography were also included in the analysis.

\section{Acute vertebral compression fracture defined using magnetic resonance imaging}

A 1.5-T magnetic resonance imaging (MRI) scanner (Achieva 1.5T Nova Dual; Philips Electronics Japan, Tokyo, Japan) was used in this study. Sagittal T1-weighted (repetition time [TR], 400; echo time [TE], 14) and axial and sagittal T2-weighted fast spin-echo (TR, 4,000; TE, 102) sequences were obtained using a $256 \times 256$ matrix, 260-mm field of view, and 3-mm/1-mm slice thickness/

Table 1. Physical examinations, TBS, BMD, and skeletal muscle mass, in the patient participants with vertebral compression fracture and control group

\begin{tabular}{|lccc}
\hline Characteristic & VCF & Non-VCF & $p$-value \\
\hline Physical examinations & & & \\
\hline No. of subjects & 43 & 99 & \\
\hline Age $(\mathrm{yr})$ & $79 \pm 6.8$ & $70 \pm 9.9$ & $<0.05$ \\
\hline Height $(\mathrm{cm})$ & $148.8 \pm 7.9$ & $152.9 \pm 7.3$ & 0.001 \\
\hline Weight $(\mathrm{kg})$ & $47.5 \pm 8.9$ & $53 \pm 6.8$ & $<0.05$ \\
\hline Body mass index $\left(\mathrm{kg} / \mathrm{m}^{2}\right)$ & $21.4 \pm 3.5$ & $22.8 \pm 3.2$ & 0.003 \\
\hline Steroid users & $14.0(6 / 43)$ & $5.0(4 / 99)$ & $<0.05$ \\
\hline Patients with rheumatic arthritis & $14.0(6 / 43)$ & $7.0(7 / 99)$ & 0.19 \\
\hline Patients with diabetes mellitus & $14.0(6 / 43)$ & $8.0(8 / 99)$ & 0.28 \\
\hline Prevalence of sarcopenia & $70.0(30 / 43)$ & $47.4(47 / 99)$ & $<0.05$ \\
\hline TBS & $1.25 \pm 0.1$ & $1.29 \pm 0.1$ & 0.02 \\
\hline BMD & & & \\
\hline Lumbar $\left(\mathrm{g} / \mathrm{cm}^{2}\right)$ & $0.76 \pm 0.2$ & $0.89 \pm 0.2$ & $<0.05$ \\
\hline Femoral $\left(\mathrm{g} / \mathrm{cm}^{2}\right)$ & $0.50 \pm 0.1$ & $0.58 \pm 0.1$ & $<0.05$ \\
\hline Skeletal muscle mass & & & \\
\hline Skeletal muscle mass index $\left(\mathrm{kg} / \mathrm{m}^{2}\right)$ & $5.07 \pm 0.1$ & $5.58 \pm 0.1$ & $<0.05$ \\
\hline Lean mass arm $(\mathrm{kg})$ & $2.70 \pm 0.5$ & $3.20 \pm 1.9$ & 0.01 \\
\hline Lean mass leg $(\mathrm{kg})$ & $8.60 \pm 1.7$ & $9.90 \pm 1.5$ & $<0.05$ \\
\hline Lean mass trunk $(\mathrm{kg})$ & $14.8 \pm 0.9$ & $16.5 \pm 2.1$ & $<0.05$ \\
\hline Vas & & & \\
\hline
\end{tabular}

Values are presented as number, mean \pm standard deviation, or \% (number/total number).

TBS, trabecular bone score; BMD, bone mineral density; VCF, vertebral compression fracture. gap. An acute VCF was diagnosed when clinical symptoms such as acute back pain were reported, and the MRI showed a low-intensity T1-weighted image and a highintensity T2-weighted image in the lumbar vertebral body. Diagnoses were made by two spine surgeons (S.T. and Y.E.) whose combined experience exceeds 10 years.

\section{Measurement of bone mineral density and skeletal muscle mass}

Body composition was measured using whole-body DXA (Hologic QDR-DELPHIW scanner DPX-NT; Hologic, Waltham, MA, USA). This DXA system provided the mass of lean soft tissue, fat, and bone mineral content for both the whole body and specific regions of the body, such as the upper extremities, lower extremities, and trunk. Appendicular skeletal muscle mass was calculated as the sum of the skeletal muscle mass in the upper extremities and lower extremities, assuming that the mass of lean soft tissue was that of the skeletal muscle. The appendicular skeletal muscle mass index (SMI) was determined as the sum of the upper and lower extremity lean mass $(\mathrm{kg}) /$ height $\left(\mathrm{m}^{2}\right)$. Sarcopenia was defined as an appendicular SMI value of $<5.46 \mathrm{~kg} / \mathrm{m}^{2}$ in women based on normative data for sarcopenia in Japanese men and women [13]. The age, height, weight, body mass index (BMI), BMD, lean mass of the upper extremities, lean mass of the lower extremities, lean mass of the trunk, appendicular lean mass, and total lean mass were recorded for all patients (Table 1).

\section{Measurement of trabecular bone score}

The TBS was measured using optional DXA software (TBS iNsight v3.0; Medimaps, Canéjan, France). Raw DXA images of each lumbar spine were uploaded into the TBS iNsight software (Medimaps). Using the patented algorithm, the lumbar spine TBS was then measured in the same regions used for the lumbar spine BMD evaluations (i.e., masking of the region of interest and edge detection were copied from the DXA scans), and TBS was calculated as the mean value of the individual measurements for vertebrae L2-L4 [18].

\section{Statistical analyses}

The height, weight, BMI, BMD, appendicular lean mass, total lean mass, SMI, and TBS were measured in partici- 
pants in both groups (Table 1). Statistical analyses were performed using SAS for Windows ver. 9.4 (SAS Institute Inc., Cary, NC, USA). For each variable, the differences between groups were evaluated using an unpaired $t$-test. Differences in the number of steroid users and those with rheumatoid arthritis or diabetes mellitus and the prevalence of sarcopenia between groups were evaluated using a chi-square test. Pearson correlation coefficients were calculated to determine the correlations between appendicular SMI, TBS, and lumbar BMD in both groups. To determine whether the loss of skeletal muscle mass or sarcopenia independently predicted the occurrence of VCFs, multiple logistic regression analyses was performed using VCF as a dependent variable with skeletal muscle mass. Other covariates included in these multivariate analyses were age, lumbar BMD, femoral BMD, and TBS, all of which significantly differed between the groups. All data are expressed as the mean \pm standard deviation. A $p<0.05$ was considered statistically significant.

\section{Results}

In the VCF group, the number of vertebral fractures by vertebral level indicated the prevalence of vertebral fractures at T12-L2, the thoracolumbar junction (Fig. 2A). There were 22 patients with a single vertebral fracture, 13 patients with two vertebral fractures, and eight patients with three or more vertebral fractures. Single fractures were the most common fracture in the VCF group (Fig. 2B). A total of 21 patients had acute VCFs, and 22 patients had chronic VCFs.

The following findings significantly differed $(p<0.05)$ between the VCF group and the non-VCF group: age (79 versus 70 years, respectively), height (148.8 versus 152.9 $\mathrm{cm}$, respectively), weight (47.5 versus $53.0 \mathrm{~kg}$, respectively), BMI (21.4 versus $22.8 \mathrm{~kg} / \mathrm{m}^{2}$, respectively), femoral BMD (0.50 versus $0.58 \mathrm{~g} / \mathrm{cm}^{2}$, respectively), lumbar spine BMD (0.76 versus $0.89 \mathrm{~g} / \mathrm{cm}^{2}$, respectively), TBS (1.25 versus 1.29 , respectively), upper limb muscle mass (2.7 versus $3.2 \mathrm{~kg}$, respectively), lower limb muscle mass (8.6 versus $9.9 \mathrm{~kg}$, respectively), trunk muscle mass (14.8 versus $9.9 \mathrm{~kg}$, respectively), SMI (5.07 versus $5.58 \mathrm{~kg} / \mathrm{m}^{2}$, respectively), history of oral steroid use ( $n=6$ versus $n=4$, respectively), and prevalence of sarcopenia ( $70.0 \%$ versus $47.4 \%$, respectively). The number of patients with rheumatoid arthritis ( $\mathrm{n}=6$ versus $\mathrm{n}=7$, respectively; $p=0.19$ ) and type 2 diabetes mellitus ( $n=6$ versus $n=8$, respectively;
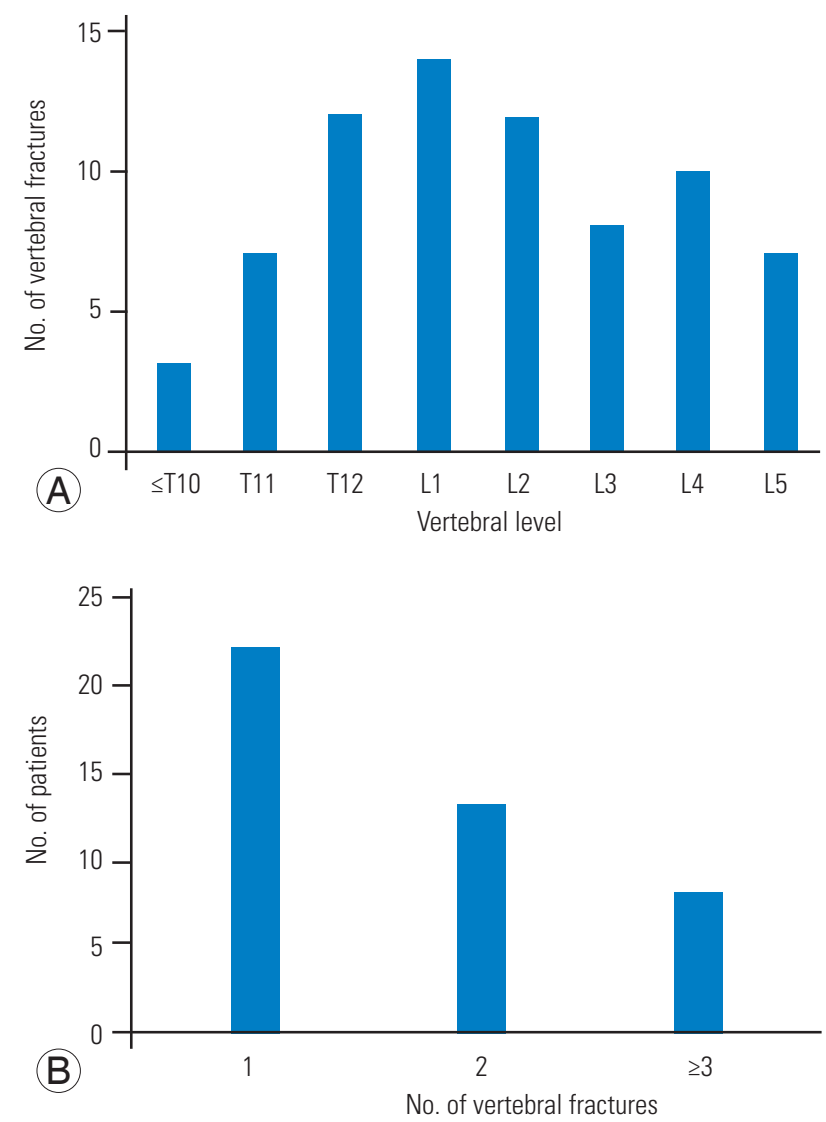

Fig. 2. The number of vertebral fractures by vertebral level in the VCF group (A) and the number of patients by vertebral fracture number in the VCF group (B). VCF, vertebral compression fracture.

$p=0.28$ ) was similar between the groups.

The VCF group was significantly older and shorter, weighed less, and had a lower BMI, BMD, and TBS, less skeletal muscle mass, and a longer history of steroid use than the non-VCF group. The prevalence of sarcopenia was significantly higher in the VCF group (70.0\%) than in the non-VCF group (47.4\%) (Table 1). BMD was positively correlated with the TBS (VCF group: $r=0.54, p<0.01$; non-VCF group: $r=0.58, p<0.01$ ) and SMI (VCF group: $r=0.20, p=0.02$; non-VCF group: $r=0.16, p<0.05)$ in both groups. The TBS was not correlated with SMI in either group (Figs. 3, 4).

The physical findings did not significantly differ between patients with acute VCFs and patients with chronic VCFs. Based on multiple logistic regression analyses, femur BMD $(p<0.01)$ and lower extremity muscle mass $(p<0.05)$ were identified as age-independent risk factors for VCF. In contrast, the TBS was not determined to be a significant explanatory factor $(p=0.90)$ (Table 2$)$. 

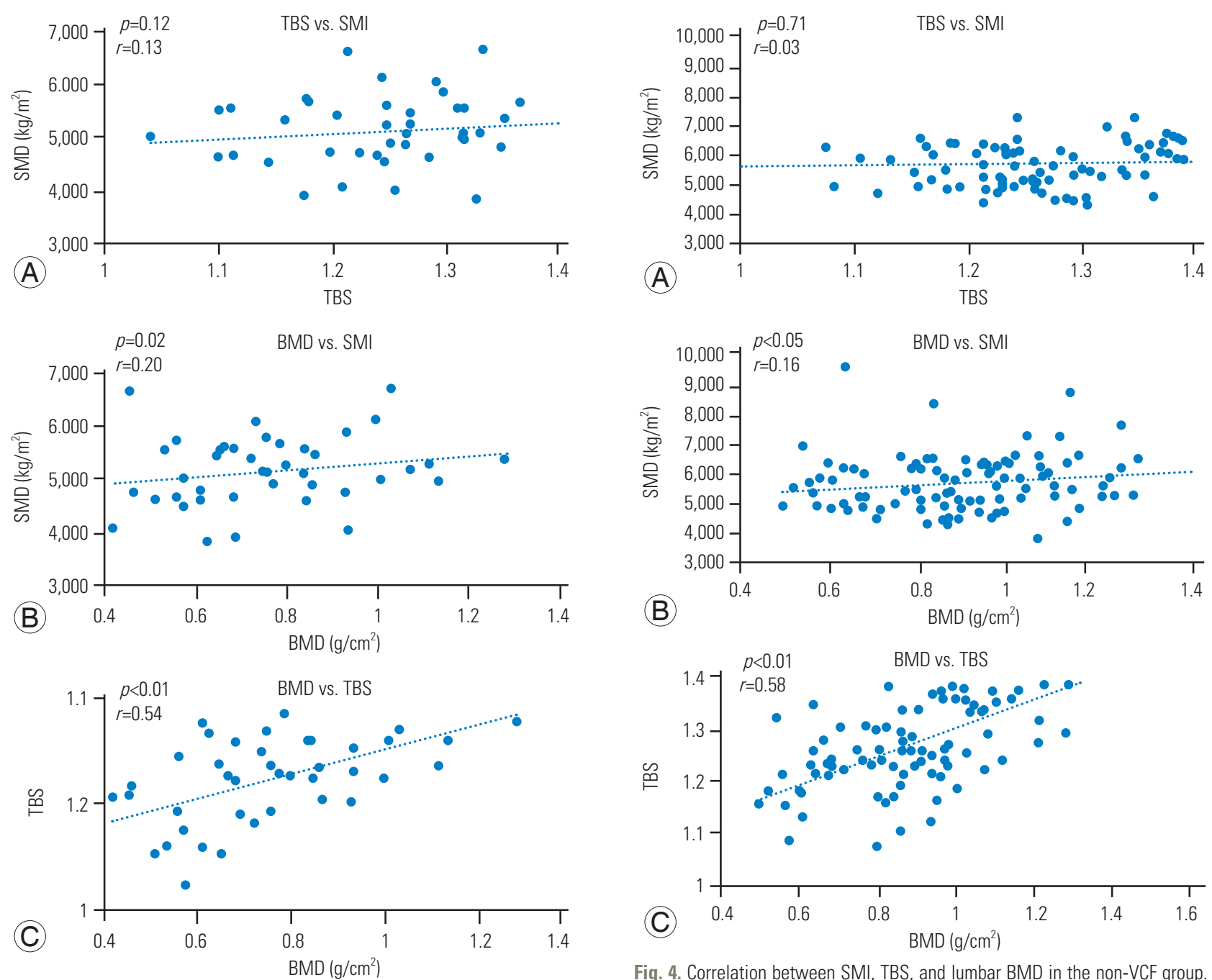

Fig. 4. Correlation between SMI, TBS, and lumbar BMD in the non-VCF group. (A) TBS vs. SMI $(r=0.03, p=0.71)$. (B) BMD vs. SMI $(r=0.16, p<0.05)$. (C) TBS vs. BMD $(r=0.58, p<0.01)$. BMD was positively correlated with the TBS and SMI, but the TBS was not correlated with SMI in the non-VCF group. SMI, skeletal muscle mass index; TBS, trabecular bone score; BMD, bone mineral density; VCF, vertebral compression fracture. muscle mass index; TBS, trabecular bone score; BMD, bone mineral density; VCF, vertebral compression fracture.

Table 2. Multiple logistic regression analysis: factors associated with the occurrence of a vertebral compression fracture

\begin{tabular}{lccc} 
Variable & B & $\chi^{2}$ value & $p$-value \\
\hline Age $(\mathrm{yr})$ & 0.107 & 8.457 & $<0.05$ \\
Lumbar BMD $\left(\mathrm{g} / \mathrm{cm}^{2}\right)$ & -2.914 & 1.196 & $<0.05$ \\
\hline Femur BMD $\left(\mathrm{g} / \mathrm{cm}^{2}\right)$ & -2.430 & 2.047 & 0.15 \\
\hline Trabecular bone score & 0.381 & 0.016 & 0.90 \\
\hline Arm muscle mass $(\mathrm{kg})$ & -2.089 & 0.213 & 0.64 \\
Leg muscle mass $(\mathrm{kg})$ & -0.001 & 0.386 & $<0.05$ \\
\hline Trunk muscle mass $(\mathrm{kg})$ & 2.770 & 0.215 & 0.21 \\
\hline B & & &
\end{tabular}

$\mathrm{BMD}$, bone mineral density.

\section{Discussion}

In this study, we investigated the relationships between skeletal muscle mass, BMD, and TBS in patients with osteoporotic VCFs. The VCF group was significantly older and had decreased femur BMD and decreased leg muscle mass compared to the non-VCF group. From the multiple logistic regression analyses, lower femoral bone density and reduced leg muscle mass were identified as risk factors for vertebral fracture independent of age, but the TBS was not identified as a risk factor.

Eguchi et al. [15] reported that reduced trunk muscle 
mass is a risk factor for degenerative scoliosis, and reduced leg muscle mass and reduced grip strength are risk factors for VCFs in elderly women [16]. Similarly, Hida et al. [19] reported that sarcopenia, low BMD, and decreased leg muscle mass were risk factors for VCFs. Decreased limb muscle mass and low femur BMD were risk factors for VCFs in this study, and these results are consistent with the results of previous studies.

The TBS is a textural index that can indirectly estimate bone microarchitecture by assessing pixel gray-level variations in lumbar DXA images. It is independent of BMD in primary osteoporosis and compliments BMD and clinical risk factors $[4,20,21]$. Reportedly, the accuracy of fracture prediction increased when the fracture risk was evaluated with the addition of the TBS to the Fracture Risk Assessment Tool, an assessment instrument used to predict fracture risk in middle-aged and elderly patients $[6,12,22,23]$.

Bilezikian et al. [24] reported that 24 weeks of treatment with subcutaneous abaloparatide, a parathyroid hormonerelated protein analog, improved the TBS in elderly women with osteoporosis. Saang et al. [25] reported that 36 months of teriparatide treatment improved the TBS in patients with secondary osteoporosis due to steroid therapy, but alendronate did not improve the TBS. According to Krieg et al. [18], 534 patients with primary osteoporosis who received bisphosphonate therapy showed improvement in their BMD and TBSs. A report from McClung et al. [26] indicated that 157 patients with osteoporosis who received denosumab for 36 months exhibited improved the TBS independent of BMD. These results illustrate that osteoporosis medications improve the TBS. However, Leslie et al. [27] revealed that improvements in the TBS using bone resorption suppression therapy did not predict fracture risk in a subanalysis of the Manitoba cohort; they disagreed with using changes in the TBS to monitor the effects of treatments for osteoporosis.

In this study, the TBS was significantly lower in the VCF group than in the non-VCF group, but it was not identified as an explanatory factor for vertebral fracture in the logistic regression analyses.

McCloskey et al. [22] reported that a TBS $\geq 1.31$ denotes a low risk for osteoporotic fractures; a TBS $>1.23$ and $<1.31$ denotes a moderate risk; and a TBS $\geq 1.23$ denotes a high risk. In the present study, the TBS was 1.25 in the VCF group, which indicated a moderate-to-high fracture risk. In the non-VCF group, the TBS was 1.29 , which indicated a low-to-moderate fracture risk and is comparable to values in previous studies.

In this study, BMD was positively correlated with the TBS and SMI in both groups, but the TBS was not correlated with SMI. Eguchi et al. [28] previously reported that both lumbar BMD and femur BMD were positively correlated with appendicular muscle mass in 971 women with and without degenerative lumbar scoliosis, which is consistent with the results in the present study. These results indicate that skeletal muscle mass correlates with BMD but not the TBS.

The present study has several limitations: (1) a small number of participants were investigated, which necessitates confirmation of our findings in a larger population; (2) the study design was cross-sectional and not longitudinal; (3) the TBS was limited to the lumbar spine, and femoral TBS will likely be required to evaluate femoral bone quality in the future; and (4) the evaluation was only for muscle mass, and an evaluation of muscle strength, such as grip strength, is also warranted.

\section{Conclusions}

The VCF group was significantly older and shorter and had a lower body weight, BMI, BMD, TBS and less skeletal muscle mass than the non-VCF group. Based on the multiple logistic regression analyses, lower femoral BMD and decreased leg muscle mass were age-independent explanatory risk factors for vertebral fracture, but the TBS was not.

\section{Conflict of Interest}

No potential conflict of interest relevant to this article was reported.

\section{References}

1. Sakuma M, Endo N, Oinuma T, et al. Incidence and outcome of osteoporotic fractures in 2004 in Sado city, Niigata prefecture, Japan. J Bone Miner Metab 2008;26:373-8.

2. Hans D, Goertzen AL, Krieg MA, Leslie WD. Bone microarchitecture assessed by TBS predicts osteoporotic fractures independent of bone density: the Manitoba study. J Bone Miner Res 2011;26:2762-9.

3. Pothuaud L, Barthe N, Krieg MA, Mehsen N, Carceller P, Hans D. Evaluation of the potential use of 
trabecular bone score to complement bone mineral density in the diagnosis of osteoporosis: a preliminary spine BMD-matched, case-control study. J Clin Densitom 2009;12:170-6.

4. Silva BC, Leslie WD, Resch H, et al. Trabecular bone score: a noninvasive analytical method based upon the DXA image. J Bone Miner Res 2014;29:518-30.

5. Shevroja E, Lamy O, Kohlmeier L, Koromani F, Rivadeneira F, Hans D. Use of Trabecular Bone Score (TBS) as a complementary approach to dualenergy X-ray absorptiometry (DXA) for fracture risk assessment in clinical practice. J Clin Densitom 2017;20:334-45.

6. Chuang MH, Chuang TL, Koo M, Wang YF. Trabecular bone score reflects trabecular microarchitecture deterioration and fragility fracture in female adult patients receiving glucocorticoid therapy: a pre-post controlled study. Biomed Res Int 2017;2017:4210217.

7. Paggiosi MA, Peel NF, Eastell R. The impact of glucocorticoid therapy on trabecular bone score in older women. Osteoporos Int 2015;26:1773-80.

8. Kim D, Cho SK, Kim JY, Choi YY, Sung YK. Association between trabecular bone score and risk factors for fractures in Korean female patients with rheumatoid arthritis. Mod Rheumatol 2016;26:540-5.

9. Breban S, Briot K, Kolta S, et al. Identification of rheumatoid arthritis patients with vertebral fractures using bone mineral density and trabecular bone score. J Clin Densitom 2012;15:260-6.

10. Dhaliwal R, Cibula D, Ghosh C, Weinstock RS, Moses AM. Bone quality assessment in type 2 diabetes mellitus. Osteoporos Int 2014;25:1969-73.

11. Leslie WD, Aubry-Rozier B, Lamy O, Hans D; Manitoba Bone Density Program. TBS (trabecular bone score) and diabetes-related fracture risk. J Clin Endocrinol Metab 2013;98:602-9.

12. Janssen I, Baumgartner RN, Ross R, Rosenberg IH, Roubenoff R. Skeletal muscle cutpoints associated with elevated physical disability risk in older men and women. Am J Epidemiol 2004;159:413-21.

13. Sanada K, Miyachi M, Tanimoto M, et al. A crosssectional study of sarcopenia in Japanese men and women: reference values and association with cardiovascular risk factors. Eur J Appl Physiol 2010;110:5765.

14. Sjoblom S, Suuronen J, Rikkonen T, Honkanen R, Kroger H, Sirola J. Relationship between postmeno- pausal osteoporosis and the components of clinical sarcopenia. Maturitas 2013;75:175-80.

15. Eguchi Y, Suzuki M, Yamanaka H, et al. Associations between sarcopenia and degenerative lumbar scoliosis in older women. Scoliosis Spinal Disord 2017;12:9.

16. Eguchi Y, Toyoguchi T, Orita S, et al. Reduced leg muscle mass and lower grip strength in women are associated with osteoporotic vertebral compression fractures. Arch Osteoporos 2019;14:112.

17. Yoshimura N, Kinoshita H, Danjoh S, et al. Prevalence of vertebral fractures in a rural Japanese population. J Epidemiol 1995;5:171-5.

18. Krieg MA, Aubry-Rozier B, Hans D, Leslie WD; Manitoba Bone Density Program. Effects of antiresorptive agents on trabecular bone score (TBS) in older women. Osteoporos Int 2013;24:1073-8.

19. Hida T, Shimokata H, Sakai Y, et al. Sarcopenia and sarcopenic leg as potential risk factors for acute osteoporotic vertebral fracture among older women. Eur Spine J 2016;25:3424-31.

20. Rabier B, Heraud A, Grand-Lenoir C, Winzenrieth R, Hans D. A multicentre, retrospective case-control study assessing the role of trabecular bone score (TBS) in menopausal Caucasian women with low areal bone mineral density (BMDa): analysing the odds of vertebral fracture. Bone 2010;46:176-81.

21. Iki M, Tamaki J, Kadowaki E, et al. Trabecular bone score (TBS) predicts vertebral fractures in Japanese women over 10 years independently of bone density and prevalent vertebral deformity: the Japanese Population-Based Osteoporosis (JPOS) cohort study. J Bone Miner Res 2014;29:399-407.

22. McCloskey EV, Oden A, Harvey NC, et al. A metaanalysis of trabecular bone score in fracture risk prediction and its relationship to FRAX. J Bone Miner Res 2016;31:940-8.

23. Silva BC, Leslie WD. Trabecular bone score: a new DXA-derived measurement for fracture risk assessment. Endocrinol Metab Clin North Am 2017;46:153-80.

24. Bilezikian JP, Hattersley G, Fitzpatrick LA, et al. Abaloparatide-SC improves trabecular microarchitecture as assessed by trabecular bone score (TBS): a 24-week randomized clinical trial. Osteoporos Int 2018;29:323-8.

25. Saag KG, Agnusdei D, Hans D, et al. Trabecular bone 
score in patients with chronic glucocorticoid therapy-induced osteoporosis treated with alendronate or teriparatide. Arthritis Rheumatol 2016;68:2122-8.

26. McClung MR, Lippuner K, Brandi ML, et al. Effect of denosumab on trabecular bone score in postmenopausal women with osteoporosis. Osteoporos Int 2017;28:2967-73.
27. Leslie WD, Majumdar SR, Morin SN, Hans D, Lix LM. Change in Trabecular Bone Score (TBS) with antiresorptive therapy does not predict fracture in women: the Manitoba BMD cohort. J Bone Miner Res 2017;32:618-23.

28. Eguchi $\mathrm{Y}$, Toyoguchi T, Inage $\mathrm{K}$, et al. Analysis of skeletal muscle mass in women over 40 with degenerative lumbar scoliosis. Eur Spine J 2019;28:1618-25. 